\title{
Situación actual de la interpretación sanitaria en la isla de Gran
}

Canaria

\section{Current situation of healthcare interpreting in Gran Canaria (Spain)}

Jessica Pérez-Luzardo Díaz ${ }^{1}$ (i) https://orcid.org/0000-0002-2748-9892

Universidad de Las Palmas de Gran Canaria

Calle Pérez del Toro, $1 \cdot 35003 \cdot$ Las Palmas de Gran Canaria · Spain

Mónica del Carmen Santana García (D) https://orcid.org/0000-0003-3389-455X

Universidad de Las Palmas de Gran Canaria

Calle Pérez del Toro, $1 \cdot 35003 \cdot$ Las Palmas de Gran Canaria · Spain

\section{AbStRACT}

The main aim of this study is to discover communication problems between healthcare staff and foreign patients at public health centres in Gran Canaria (Spain). We define healthcare interpreting after framing it within bilateral interpreting and community interpreting. We then address the situation of healthcare interpreting in Spain (specifically, in the Canary Islands), which differs from that of other countries. We outline an observational descriptive study carried out through surveys among health and non-health personnel (office workers and orderlies) at public hospitals in Gran Canaria. The results show the extent of the communication problem at the different hospital services under study and the ad hoc solutions applied in most cases by health professionals themselves, as well as the almost total absence of professional interpreters.

Key words: interpreting, healthcare interpreting, communication problems, professional interpreters

\section{Resumen}

El objetivo principal de este estudio es averiguar los problemas de comunicación que existen entre el personal sanitario y el paciente extranjero en los centros sanitarios públicos de la isla de Gran Canaria. Tras enmarcar la interpretación sanitaria dentro de la interpretación bilateral y de la interpretación en los Servicios Públicos, se define la interpretación sanitaria y se aborda la situación de esta en España y, en concreto, en Canarias, que difiere de la profesión en otros países. Se explica un estudio observacional descriptivo realizado mediante encuestas entre el personal sanitario y no sanitario (administrativos y celadores) de los complejos hospitalarios públicos de Gran Canaria. Se analizan los resultados obtenidos, que ponen de manifiesto la dimensión del problema de comunicación que existe en los diferentes servicios de los complejos hospitalarios en estudio y las soluciones ad hoc que se aplican en la mayoría de los casos a cargo del propio personal sanitario, así como la ausencia casi total de profesionales de la interpretación.

Palabras clave: interpretación sanitaria, barreras comunicativas, interpretación profesional vs interpretación ad hoc 


\section{Introducción}

La interpretación existe desde tiempos inmemoriales y es con probabilidad una de las actividades más antiguas (Jiménez Ivars, 2012). Aunque «se ignora, porque la voz no deja huellas materiales, cuándo surgió el lenguaje oral o a qué se debió su aparición» (ibídem, 2012:41), esta actividad es muy posible que surgiera cuando las relaciones entre grupos con lenguas diferentes se intensificaron, y ya no era suficiente el gesto para poder comunicarse. Es por eso por lo que hay multitud de definiciones. Pöchhacker (2004) define la interpretación como una forma de traducción «in which a first and final rendition in another language is produced on the basis of a one-time presentation of an utterance in a source language» (2004:11). Se trata de traducir un mensaje en una lengua de origen que solo se puede escuchar una vez en la lengua original, y reproducirlo en la lengua meta sin la oportunidad de volver a reformular el mensaje. Esta definición se refiere especialmente a la interpretación simultánea y la interpretación consecutiva, pero también es extrapolable a otras técnicas o modalidades. El presente estudio se centra en la interpretación bilateral, técnica de interpretación más frecuente en el contexto de la interpretación sanitaria.

Las variedades de interpretación se clasifican, por un lado, teniendo en cuenta las técnicas de interpretación estas son las que hacen referencia a las operaciones mentales que realiza el intérprete y a las distintas actividades que se comportan en el proceso-, a saber: la interpretación consecutiva, la interpretación simultánea y la interpretación de enlace o bilateral (cf. Collados Aís, 2001). Y, por otro lado, según los ámbitos de interpretación, estos se pueden subdividir en dos bloques, los monológicos y los dialógicos. Los primeros se encuentran en la interpretación simultánea, la interpretación consecutiva, la interpretación susurrada y la traducción a la vista, mientras que los segundos, se encuentran en la interpretación bilateral, ya que esta se da en situación de diálogo, es decir, la interpretación se realiza entre dos hablantes que no comparten la misma lengua.

La interpretación bilateral es la técnica de interpretación más utilizada en la interpretación de los Servicios Públicos, puesto que se da en situación de diálogo entre dos participantes que no tienen una lengua en común. La interpretación sanitaria se engloba dentro de la interpretación bilateral, pero no solo se recurre a esta técnica, en menor medida también se utiliza la interpretación bilateral con toma de notas o la interpretación consecutiva.

\section{Marco teórico}

\subsection{La interpretación sanitaria}

Tras enmarcar la interpretación sanitaria dentro de la interpretación de bilateral y dentro de la interpretación de los Servicios Públicos, definiremos la interpretación sanitaria.

La interpretación sanitaria o interpretación en el ámbito sanitario es aquella que tiene lugar entre el personal sanitario y un paciente que no comparte la misma lengua. Según Hale (2012:35) esta interpretación «incluye consultas privadas, hospitales y consultas con diferentes profesionales sanitarios como logopedas, dietistas o psicoterapeutas».

Los estudios en este ámbito comienzan a cobrar fuerza a partir de los años 90. Parece que hay consenso en que el paciente que no habla el mismo idioma que el personal sanitario recibe una atención de calidad peor que si comparte el mismo idioma (cf. Hsieh, 2015). Países como Estados Unidos o Canadá han puesto en marcha un 
servicio de interpretación en los hospitales para que los pacientes extranjeros estén en las mismas condiciones que los pacientes locales o aquellos pacientes que conocen el idioma del país.

Hay evidencia científica suficiente para afirmar que la interpretación en el ámbito sanitario es un servicio esencial para poder proporcionar una atención sanitaria de calidad a aquellos pacientes que no conocen la lengua local y evitar un diagnóstico erróneo. Como explican Crezee et al (2015:13), «an incorrect diagnosis may lead to either an inappropriate course of treatment or the patient being discharged in error. This may lead to frequent re-admissions or deterioration in the patient's condition».

\subsection{La interpretación sanitaria en España}

El acceso a un servicio de traducción e interpretación en el ámbito sanitario en España es aún más un privilegio que un derecho. Para suplir la falta de un servicio de traducción e interpretación en los centros sanitarios españoles, en algunos centros de España se ha introducido el uso de la interpretación telefónica. Pero en muchas ocasiones el personal sanitario no termina de aceptar esta solución porque ven el servicio como algo impersonal y poco práctico (cf. Foulquié-Rubio et al, 2018) y en algunos casos el personal no conoce de la existencia del servicio de interpretación telefónica.

Debido a la inexistencia de un servicio de interpretación en los centros sanitarios en muchas partes de España, el personal es el que debe encontrar soluciones improvisadas (comunicación mediante gestos, participación de familiares de los pacientes haciendo las veces de intérpretes, otros compañeros e incluso traductores automáticos) para poder comunicarse con el paciente extranjero (cf. ibídem, 2018).

A continuación, comentaremos brevemente cuál es la situación actual de la interpretación sanitaria en Canarias para, posteriormente, centrarnos en la isla de Gran Canaria.

\subsection{La interpretación sanitaria en Canarias}

Las Islas Canarias son un foco de atractivo turístico. Ya en los años cincuenta, Canarias comenzó a recibir turistas interesados en curar sus problemas de salud en las Islas, en su mayoría eran alemanes. En el municipio de Firgas, se encontraba el Balneario de Azuaje con sus manantiales curativos, ricos en hierro en disolución. Frecuentaba sus instalaciones gente del norte de Europa para disfrutar del clima templado y de las aguas curativas (cf. Humberto Pérez, 2016). A continuación, se muestra una de las cartas que se enviaban al alcalde del municipio pidiendo información sobre el Balneario de Azuaje. 
25/04/1919: Gobierno Militar de Gran Canaria

Alemán enfermo con licencia para acudir a los Baños.

Para el Señor Alcalde Constitucional de Firgas.

"Debiendo marchar en breve á ese pueblo el Oficial de la Armada Alemana Señor KIRCHNER, en uso de dos meses de licencia por enfermo que le han sido concedidos por el Excmo. Señor Capitán General del distrito, acompañado de su asistente el marino BOHM, participo á V.S. que dicha superior autoridad ha dispuesto á la vez que se presenten quincenalmente en esa Alcaldía esperando tenga á bien participarme las fechas en que verifiquen las presentaciones y caso de ausentarme del mismo me dé cuenta por el medio más rápido de que disponga."

Dios guarde á V.S. muchos años.

Las Palmas 25 Abril de 1919.

Fmd.: El General Gobernador. [Ilegíble].

Extracto de Perdomo Cerpa, Manuel. 1992. Firgas. Noticias de un Siglo 1835-1935.Firgas, Gran Canaria. Estudigraf, S.L. Pp. 467.

Pero no sería hasta los años 80 y 90 cuando se desarrolló en el archipiélago el turismo de salud. Según el Ministerio de Industria, Energía y Turismo (2013) el turismo de salud es el proceso por el cual una persona recibe atención médica en un país extranjero.

En la década de los 90, en las Islas Canarias, se asentaron personas de diferentes países, debido en parte a la situación geográfica en la que se encuentra el archipiélago (cf. Toledano Buendía, 2006), pero también por su reconocido clima templado. Y algo propio de este archipiélago es que la afluencia del turismo es relativamente constante durante todo el año. Es posible distinguir dos grupos de población extranjera. Por un lado, los visitantes y turistas extranjeros «cuyos desplazamientos se caracterizan por tratarse de estancias breves, realizándose por motivos de placer y ocio»; y, por otro lado, la población extranjera propiamente dicha que se instala en la isla «por motivos tan variados como pueden ser la jubilación, la internacionalización de la economía canaria o la pobreza y los conflictos políticos de otros países» (ibídem, 2006:188). Debido al gran número de extranjeros, ya sean turistas o residentes en Canarias de uno u otro grupo, que frecuentan el archipiélago, la gran mayoría necesitan asistencia lingüística en los servicios públicos. Por esa razón, el Gobierno puso en marcha el Primer Plan Canario para la Inmigración 2002-2004 y este plan se definía de la siguiente manera (I PCI: 42):

La consideración de los/as extranjeros/as como personas titulares de derechos y deberes, en condiciones de igualdad con los/as españoles/as, eliminando toda forma de discriminación por motivos de nacionalidad, raza o creencias, particularmente en los ámbitos de las condiciones laborales, educativo, cultural, de la vivienda, la sanidad, etc., debe impregnar todas las actuaciones públicas.

Además, se elaboró un Segundo Plan Canario de Inmigración, pero, como explica Toledano Buendía (2006:195) «podemos afirmar, [...] que, lamentablemente, la mayoría de estas acciones se han quedado en meras declaraciones de intentos sin que se haya adoptado casi ninguna medida práctica».

La población extranjera (según lugar de procedencia), según el Instituto Nacional de Estadística (INE) y el Instituto Canario de Estadística (ISTAC) asciende a un total de 260.191 (véase la Tabla 1). 
Población extranjera según lugar de procedencia (01/01/2018)

\begin{tabular}{|c|c|c|c|c|c|c|c|}
\hline & Total & $\begin{array}{l}\text { Unión } \\
\text { Europea } \\
\text { (28) }\end{array}$ & $\begin{array}{l}\text { Resto } \\
\text { de Europa }\end{array}$ & África & América & Asia & Oceanía \\
\hline Canarias & 260.191 & 128.194 & 10.382 & 24.811 & 63.717 & 16.377 & 147 \\
\hline Lanzarote & 33.003 & 15.118 & 629 & 4.654 & 7.768 & 2.351 & 14 \\
\hline Fuerteventura & 32.112 & 18.342 & 499 & 4.371 & 5.522 & 1.164 & 12 \\
\hline Gran Canaria & 66.363 & 26.234 & 3.236 & 8.457 & 17.706 & 6.180 & 69 \\
\hline Tenerife & 117.196 & 61.139 & 5.607 & 7.001 & 29.900 & 6.467 & 48 \\
\hline La Gomera & 2.904 & 2.074 & 98 & 97 & 501 & 41 & 0 \\
\hline La Palma & 7.573 & 4.688 & 281 & 183 & 1.992 & 169 & 3 \\
\hline El Hierro & 1.040 & 599 & 32 & 48 & 328 & 5 & 1 \\
\hline
\end{tabular}

Tabla 1. Población extranjera según lugar de procedencia. Elaboración propia (ISTAC 2018)

Es por ello por lo que parece necesario contar con la figura de un traductor o intérprete en los Servicios Públicos, para poder brindar a los usuarios que no comparten nuestra lengua una atención de calidad e igualdad frente a los que sí la hablan.

En el ámbito sanitario en Canarias debemos hacer una distinción entre centros públicos y centros privados, puesto que los centros privados sí cuentan con intérpretes en plantilla para ayudar al personal sanitario y administrativo a comunicarse con los pacientes extranjeros. La situación en el sector público es totalmente opuesta. Antes de la crisis económica, se implantó un servicio de interpretación telefónica a los pacientes extranjeros que hacían uso del Servicio Canario de Salud (SCS). Pero esta medida no fue bien recibida por parte del personal sanitario. Se criticó que se destinara dinero al servicio de interpretación. El servicio quedó cancelado y desde entonces no queda constancia de que se proporcione asistencia lingüística (salvo algunas excepciones). Para suplir esa falta, es el propio personal el que debe tomar medidas y buscar soluciones para poder comunicarse con el paciente (cf. Pérez-Luzardo Díaz y Fernández Pérez, 2018).

Desde junio de 2017 en algunos hospitales de la provincia de Tenerife hay un servicio de interpretación telefónica en el servicio de urgencias. Sin embargo, según el estudio de Pérez-Luzardo Díaz y Fernández Pérez (2018) no hay constancia de que exista un servicio parecido en la provincia de Las Palmas. Además, el personal sanitario no tiene claro cuál es el perfil profesional del traductor e intérprete y, por consiguiente, no sabe qué esperar de este. Otro problema es que, al no estar centralizado el servicio, resulta difícil localizar a intérpretes que estén disponibles. A esto se suma que algunos no consideran que el intérprete sea necesario y alegan que «es suficiente con tener un conocimiento acreditado de las lenguas de trabajo» (ibídem, 2018:71). El SCS editó en su momento carteles en diferentes idiomas para que el paciente extranjero llevase a su propio intérprete si tenía que acudir a un centro sanitario (Toledano Buendía, et al., 2006), pero desde entonces los carteles han ido desapareciendo de los centros de salud, sin que se haya introducido ninguna novedad en el procedimiento. 
Resulta curioso que existan aplicaciones de traducción médica que han sido diseñadas para ayudar tanto al personal sanitario como al paciente extranjero. El SCS en concreto tiene disponibles dos aplicaciones gratuitas, estas son: TRADASSAN e Hipot-CNV. No obstante, su grado de implantación como veremos más adelante es muy escaso.

Como se puede apreciar en la tabla 1, las personas extranjeras frecuentan más las islas capitalinas, la isla de Tenerife y la de Gran Canaria. Podemos decir que en este estudio nos centraremos en las islas que pertenecen a la provincia de Las Palmas, es decir, a Gran Canaria, Fuerteventura y Lanzarote, ya que uno de los objetivos de este trabajo es conocer los problemas de comunicación en los dos hospitales públicos de la isla de Gran Canaria, pero estos hospitales también reciben pacientes de las islas de Fuerteventura y Lanzarote.

En el siguiente apartado nos centraremos en explicar y analizar el estudio que llevamos a cabo para averiguar los problemas de comunicación a los que se enfrenta el personal sanitario perteneciente a los dos hospitales públicos de la isla de Gran Canaria.

\section{Metodología}

El objetivo principal de este estudio es averiguar los problemas de comunicación entre el personal sanitario y el paciente extranjero en los centros sanitarios de Gran Canaria. Forma parte de un estudio más amplio en el cual se ha empleado un cuestionario que se basa en el realizado en el estudio de Pérez-Luzardo Díaz y Fernández Pérez (2018).

El cuestionario, que consta de diecinueve preguntas, se divide en tres bloques principales. El primer bloque trata sobre los datos generales sobre la población de estudio (sexo, edad, profesión, etc.). El segundo bloque aborda la recepción y la comunicación con pacientes extranjeros en los hospitales públicos de la isla de Gran Canaria, y el tercer bloque se centra en los traductores e intérpretes, es decir, se pregunta a los encuestados sobre si creen que debería existir un servicio de traducción e interpretación o qué cualificaciones creen que debería tener un traductor e intérprete sanitario.

En este trabajo se abordan las preguntas 12, 13 y 14 del cuestionario, correspondientes al segundo bloque del cuestionario. En la pregunta 12 se pide a los encuestados que indiquen si han experimentado problemas de comunicación con los usuarios extranjeros. Se trata de una variable estadística categórica, por lo que no se calculan medias y desviaciones típicas. En la pregunta 13 se ahonda en la cuestión, al pedirles que en caso de haber respondido afirmativamente a la pregunta anterior, indiquen qué problemas han surgido. Para responder se les presenta un listado de opciones y se les abre la posibilidad de añadir una opción adicional. En este estudio nos centramos en el primer problema «no hablaban comprensiblemente el idioma». En la pregunta 14, nos interesa averiguar cómo se ha abordado el problema, es decir, cómo se ha resuelto. También en esta ocasión, presentamos a los sujetos del estudio una batería de soluciones y damos la oportunidad de que añadan otras opciones no previstas por el cuestionario. Dado que las opciones son múltiples y en este estudio nos centramos en la correlación entre el problema «no hablaban comprensiblemente el idioma» con las diferentes opciones de respuesta a la pregunta 14, para cada variable podrá haber menos respuestas que el tamaño de la muestra.

La muestra total del estudio es de 289 personas, de las cuales 220 son mujeres $(76,12 \%)$ y 69 son hombres $(23,88 \%)$, todos vinculados a uno de los dos hospitales públicos de Gran Canaria (140, Complejo Hospitalario Universitario Insular-Materno Infantil y 149, Hospital Universitario de Gran Canaria Doctor Negrín). El rango de edad mejor representado es el de 41-50 (37,72\%). 179 de los encuestados $(62,94 \%)$ tiene una antigüedad 
superior a 13 años. En cuanto a la lengua materna, 280 tienen español como lengua materna (96,89\%) y 193 consideran que hablan inglés (84\%), pero no todos tienen un nivel acreditado del idioma.

Los sujetos representan a los distintos perfiles profesionales del entorno hospitalario, siendo el cuerpo de enfermeros el más representado con un 49,13\% del total (142 sujetos), seguido por los auxiliares de enfermería (24,91\% del total, 72 sujetos), y un $13.49 \%$ de médicos (39 sujetos). Los celadores (5,54\%), el cuerpo administrativo $(4,15 \%)$ y el personal técnico $(2,77 \%)$ también están representados, aunque en menor medida. Esta distribución representa a una pequeña parte de la población de ambos complejos hospitalarios en cuestión. La muestra se centra en aquellos perfiles que mayor contacto con el paciente tienen, en concreto, distribuimos los cuestionarios en los siguientes servicios:

- Urgencias

- Consultas externas

- Quirófano

- Áreas de hospitalización (traumatología, obstetricia, neonatos...)

- Áreas especiales (radiología vascular, exploraciones especiales de urología...)

- Radiología

- Administración

Hemos elegido estos servicios dentro del hospital porque son las posibles áreas donde el paciente llega en primer lugar al centro hospitalario y requiere de atención médica, y donde puede permanecer ingresado tras alguna intervención o prueba médica.

El procedimiento que utilizamos para recabar los datos fue asistir en persona a los servicios anteriormente mencionados y contactar con el jefe de servicio o supervisor para informarle de nuestro estudio y para que, con su colaboración, el personal de cada servicio cumplimentase el cuestionario. Proporcionamos copias del cuestionario a los jefes de servicio o supervisores, quienes se encargaron de hacer llegar el cuestionario al personal de su servicio, colaboración con la que estamos muy agradecidas. El cuestionario estuvo disponible en cada servicio una semana, es decir, se entregaron a comienzo de semana y se recogió al final de esa misma semana. Tuvimos casos en los que al recogerlos el supervisor nos pedía un poco más de tiempo para que todos estuvieran cumplimentados. Además, otros nos pidieron que dicho cuestionario lo pasáramos por correo electrónico, por lo tanto, elaboramos un formulario de Google y compartimos el cuestionario también por esa vía.

Para el estudio estadístico se tabularon las respuestas en una hoja Excel, se procedió a la validación y depuración de los datos, se codificaron las categorías de cada variable, las variables con respuesta múltiple se desglosaron en varias variables para cada respuesta, se generó una nueva variable con todas las respuestas, por lo que pueden tener más respuestas que elementos en la muestra. Para este caso concreto, en el que se estudia la relación entre dos variables, se ha realizado una tabla de contingencia que se expone a continuación. Para el estudio estadístico se usó el software libre RStudio. Se trata de un estudio de estadística descriptiva, que se obtiene directamente de los datos, de ahí su validez. Para realizar el estudio de Fisher necesitaríamos conocer el dato correspondiente a 
la población total de personas extranjeras que fueron a los hospitales durante la recogida de datos, algo fuera de nuestro alcance al no existir tal registro.

\section{Resultados}

A la primera pregunta (Indique si ha experimentado problemas de comunicación con los usuarios extranjeros) el 91,00\% de los sujetos encuestados afirma que ha experimentado problemas de comunicación en el desempeño de su trabajo, frente al 5,88\% que afirma que nunca ha experimentado problemas de comunicación. Este resultado realmente pone de manifiesto la dimensión del problema que estamos abordando.

En la siguiente pregunta recordamos que ofrecemos a los sujetos un abanico de problemas de comunicación. Debido a la extensión del estudio, en este trabajo nos centramos en el que consideramos principal problema de comunicación que sería cuando los pacientes no hablan comprensiblemente el idioma. Resulta llamativo que tan solo 27 sujetos $(10,47 \%)$ de los 289 participantes haya señalado "pocas veces", el resto $(89,53 \%)$ señala que los problemas de comunicación se deben a que los pacientes no hablan comprensiblemente el idioma con cierta $(35,27 \%)$, bastante $(35,27 \%)$ o con mucha frecuencia $(18,99 \%)$. Ante esta manifiesta frecuencia en los problemas de comunicación con pacientes que no se comunican lo suficiente en español, cobra relevancia analizar qué estrategias o soluciones aborda el personal sanitario para poder comunicarse correctamente. En la última pregunta de este análisis indagamos precisamente en las soluciones que representamos en la siguiente tabla (véase tabla 2).

\begin{tabular}{|c|c|c|c|c|}
\hline $\begin{array}{l}\text { Tipo de solución al problema de } \\
\text { comunicación }\end{array}$ & $\begin{array}{l}\text { Pocas veces } \\
\quad(\%)\end{array}$ & $\begin{array}{c}\text { Con cierta } \\
\text { frecuencia }(\%)\end{array}$ & $\begin{array}{l}\text { Con bastante } \\
\text { frecuencia }(\%)\end{array}$ & $\begin{array}{c}\text { Con mucha } \\
\text { frecuencia }(\%)\end{array}$ \\
\hline Por gestos & $\begin{array}{c}55 \\
(21,65 \%)\end{array}$ & $\begin{array}{c}94 \\
(37,01 \%)\end{array}$ & $\begin{array}{c}70 \\
(27,56 \%)\end{array}$ & $\begin{array}{c}35 \\
(13,78 \%)\end{array}$ \\
\hline Recurriendo a un compañero & $\begin{array}{c}32 \\
(12,26 \%)\end{array}$ & $\begin{array}{c}86 \\
(32,95 \%)\end{array}$ & $\begin{array}{c}95 \\
(36,40 \%)\end{array}$ & $\begin{array}{c}48 \\
(18,39 \%)\end{array}$ \\
\hline Recurriendo a alguien externo & $\begin{array}{c}57 \\
(21,84 \%)\end{array}$ & $\begin{array}{c}74 \\
(28,35 \%)\end{array}$ & $\begin{array}{c}79 \\
(30,27 \%)\end{array}$ & $\begin{array}{c}51 \\
(19,54 \%)\end{array}$ \\
\hline $\begin{array}{l}\text { Pidiéndole al paciente que traiga a alguien que } \\
\text { le ayude }\end{array}$ & $\begin{array}{c}123 \\
(48,05 \%)\end{array}$ & $\begin{array}{c}59 \\
(23,05 \%)\end{array}$ & $\begin{array}{c}58 \\
(22,66 \%)\end{array}$ & $\begin{array}{c}16 \\
(6,25 \%)\end{array}$ \\
\hline $\begin{array}{l}\text { Pidiéndole al paciente que llame a alguien por } \\
\text { teléfono para que haga de intérprete }\end{array}$ & $\begin{array}{c}154 \\
(60,16 \%)\end{array}$ & $\begin{array}{c}59 \\
(23,05 \%)\end{array}$ & $\begin{array}{c}30 \\
(11,72 \%)\end{array}$ & $\begin{array}{c}13 \\
(5,08 \%)\end{array}$ \\
\hline $\begin{array}{l}\text { Recurriendo al Traductor de Google u otro } \\
\text { traductor automático }\end{array}$ & $\begin{array}{c}117 \\
(45,53 \%)\end{array}$ & $\begin{array}{c}62 \\
(24,12 \%)\end{array}$ & $\begin{array}{c}49 \\
(19,07 \%)\end{array}$ & $\begin{array}{c}29 \\
(11,28 \%)\end{array}$ \\
\hline $\begin{array}{l}\text { Recurriendo a aplicaciones de traducción } \\
\text { médica (TRADASSAN, HIPOT-CNV...) }\end{array}$ & $\begin{array}{c}206 \\
(81,75 \%)\end{array}$ & $\begin{array}{c}27 \\
(10,71 \%)\end{array}$ & $\begin{array}{c}14 \\
(5,56 \%)\end{array}$ & $\begin{array}{c}5 \\
(1,98 \%)\end{array}$ \\
\hline Contratando a un intérprete (lengua hablada) & $\begin{array}{c}208 \\
(83,53 \%)\end{array}$ & $\begin{array}{c}19 \\
(7,63 \%)\end{array}$ & $\begin{array}{c}14 \\
(5,62 \%)\end{array}$ & $\begin{array}{c}8 \\
(3,21 \%)\end{array}$ \\
\hline
\end{tabular}

Tabla 2. Soluciones al problema de comunicación (no hablan comprensiblemente el idioma) 
De la tabla 2 se desprende que las tres soluciones más frecuentes son llamar a un compañero que conozca el idioma del paciente, llamar a alguien externo (algún familiar) que pueda comunicarse con el personal sanitario o recurrir al lenguaje no verbal (gestos). Le siguen las opciones «recurriendo al Traductor de Google u otro traductor automático»y «pedirle al paciente que traiga a alguien que le ayude».

Más de la mitad de los participantes, 143 de los 289 sujetos, que supone el 54,79\% del total reconoce llamar a algún compañero que conozca o entienda el idioma del paciente con bastante o con mucha frecuencia. Si a esta cifra sumamos a aquellos que señalan que con cierta frecuencia recurren a esta solución alcanzamos un total de 229 , cerca del $90 \%$ de la muestra $(87,74 \%)$. Sorprende constatar que el personal pida ayuda a sus compañeros, porque cabe esperar que estos no siempre estén disponibles al tener su propio servicio que cubrir. Sin embargo, que recurran a algún familiar viene a confirmar lo que ya sabemos que ocurre tanto en el ámbito sanitario, como en otros entornos de los servicios públicos (Antonini 2015). De los 289 sujetos, 130 reconocen buscar en los familiares solución al problema comunicativo con bastante $(30,27 \%)$ o con mucha frecuencia $(19,54 \%)$.

Más de un tercio de los sujetos $(37,01 \%)$ afirma que en algún momento ha utilizado los gestos para poder comunicarse con el paciente extranjero. Otro dato curioso es que 95 de los 289 sujetos encuestados afirman que con bastante frecuencia recurren a un compañero que conozca o entienda el idioma del paciente extranjero para que la comunicación pueda tener lugar. Del mismo modo 79 de los 289 encuestados $(30,27 \%)$ afirman que suelen recurrir a alguien externo (familiar del paciente) para que se pueda comunicar con el usuario y el personal sanitario.

Resulta llamativo que el $24,12 \%$ de los encuestados afirme que con cierta frecuencia utiliza el Traductor de Google para poder comunicarse con el paciente extranjero, puesto que esta herramienta de traducción automática no es una aplicación fiable como para que el personal hospitalario se apoye en ella para poder comunicarse. El $81,75 \%$ de los sujetos encuestados no conoce la existencia de las aplicaciones de traducción médica diseñadas para comunicarse con el personal sanitario. Esto resulta muy llamativo, ya que en la misma comunidad autónoma donde se desarrolla el estudio se han desarrollado aplicaciones específicas para la comunicación con el paciente que no habla el español en el ámbito sanitario, (TRADASSAN e Hipot-CNV). Del mismo modo, es sorprendente ver que $208(83,54 \%)$ de los 289 encuestados aseguran que pocas veces o nunca han contratado a un intérprete para que les ayude a que la comunicación se lleve a cabo entre ellos y los usuarios extranjeros.

\section{Conclusiones}

Los resultados nos confirman que el personal sanitario soluciona en primera instancia los problemas de comunicación acudiendo a algún compañero que cubra con sus conocimientos de la lengua del paciente el problema tal y como ya se ha constatado en estudios anteriores (Pérez-Luzardo Díaz y Fernández Pérez, 2018). En segundo lugar, acude a alguien externo, familiares, el cónyuge, amigos o incluso menores que se ven en la tesitura de tener que mediar en situaciones comunicativas en un contexto sanitario para el que muchas veces no están preparados, ni se les puede exigir precisión ni imparcialidad (Hale 2015). Puede que desconozcan el léxico especializado, que se vean abrumados por la situación en la que se encuentra un familiar cercano, o que tergiversen por desconocimiento de las reglas del juego de la actividad que desempeñan los enunciados de unos u otros, añadiendo comentarios personales y omitiendo los que consideren superfluo o no recuerden. Que el uso del lenguaje no verbal ocupe un lugar tan destacado como estrategia de solución comunicativa no debería sorprender, pero no se escapa el peligro de la no coincidencia de gestos y expresiones entre culturas/lenguas distintas, lo que -una vez más- puede acarrear fallos en la comunicación y falsos diagnósticos. No resulta 
sorprendente tras el análisis de la situación, pero sí descorazonador, que en última instancia y casi de manera anecdótica se recurra a la figura del intérprete como mediador o solucionador de los problemas comunicativos.

Esto está relacionado directamente con la carencia de un perfil profesional, la falta del reconocimiento de la profesión de intérprete, no solo en el ámbito sanitario, sino en todos los ámbitos de los servicios públicos, que ya se ha alcanzado en otros entornos multilingües similares, como es el caso de Nueva Zelanda (cf. Crezee et al., 2015), EE.UU. (cf. Navaza et al., 2009) o en algunos países europeos como Bélgica (cf. Muñoz Martínez, 2010). La figura del intérprete en los servicios públicos contribuiría a una comunicación entre el personal de la entidad pública y el usuario extranjero. Además, brindaría un servicio de calidad y de igualdad a los pacientes o usuarios extranjeros que no hablen el español, ya que se encontrarían en las mismas condiciones que una persona local.

En la actualidad, debido a la falta de esta figura profesional, el personal sanitario es el que debe buscar soluciones por sus propios medios para poder comunicarse con un paciente extranjero. Igualmente, no todas las soluciones son igual de válidas porque, por ejemplo, no siempre se puede encontrar a un compañero que esté disponible para que le ayude o el familiar del paciente quizás no sepa adecuadamente el español, o aún peor, el familiar puede que sea un niño y este no debería de encargarse de dar malas noticias, en caso de enfermedad. También, sorprende que algunos profesionales de la sanidad utilicen el Traductor de Google para poder comunicarse con el paciente extranjero. Con los traductores automáticos pueden surgir malentendidos que provoquen diagnósticos erróneos, y por ende un mayor gasto sanitario. Una de las actividades del intérprete sanitario sería evitar ese tipo de situaciones.

Hoy en día, muchos son los que creen que para ejercer de traductor o intérprete es suficiente con conocer el idioma, pero el perfil de estos profesionales es mucho más que conocer otra lengua. Los intérpretes deben estar bien cualificados, preparados y haber recibido una buena formación previa para saber enfrentarse a todo tipo de situaciones. Por esa razón y ante la falta de intérpretes sanitarios, se debería, en primer lugar, concienciar al personal sanitario de la necesidad de esta figura y, además explicarle la función y el trabajo de los intérpretes para que sepan qué esperar de su trabajo y aprender a trabajar conjuntamente.

Para concluir, está claro que aún queda mucho por hacer y que este campo es aún desconocido para muchos. Sin embargo, deberíamos empezar reconociendo por ley que los usuarios extranjeros que hagan uso de cualquier centro sanitario deberían contar con la ayuda de un intérprete en caso de necesitarlo, como ocurre ya en otros países. Una futura línea de investigación que podría reforzar la toma de decisión para dar una solución efectiva en el ámbito sanitario de Gran Canaria sería conocer el punto de vista de los pacientes extranjeros que tienen que hacer uso de los servicios sanitarios de la isla, es decir, saber cómo se sienten cuando tienen que comunicarse con el personal sanitario que no habla su idioma. Del mismo modo, sería interesante realizar una comparación sobre los países donde la interpretación sanitaria está bien organizada, para ver qué se podría hacer en Gran Canaria para poner en marcha un servicio de interpretación sanitaria viable en los centros sanitarios públicos de la isla, una semilla para asegurar una asistencia sanitaria eficiente a aquellos ciudadanos extranjeros que lo necesiten.

\section{Declaration of conflicting interests}

The author(s) declared no potential conflicts of interest with respect to the research, authorship, and/or publication of this article. 


\section{Funding}

The author(s) received no financial support for the research, authorship, and/or publication of this article.

\section{Sobre las autoras}

Jessica Pérez-Luzardo received her Master's degree in conference interpreting at the University of La Laguna in 1997. In 2005, she obtained her PhD in Translation Studies from the University of Las Palmas de Gran Canaria with a doctoral thesis on didactics of simultaneous interpreting. Since 1996 she has taught translation and interpreting studies. Her research focuses on interpreting pre-exercises, quality parameters in conference interpreting, and affective variables such as motivation, anxiety and self-concept in the interpreting learning process.

Mónica del Carmen Santana García es Graduada en Traducción e Interpretación (inglés-francés) y máster en Traducción Profesional y Mediación Intercultural. Actualmente está terminando su proyecto de tesis doctoral que gira en torno a la interpretación sanitaria en Gran Canaria para la que disfruta de una ayuda del programa predoctoral de formación del personal investigador dentro de programas oficiales de doctorado en Canarias. Realizó su estancia de investigación en Auckland, Nueva Zelanda, para ahondar en aspectos de su tesis doctoral.

\section{Agradecimientos}

\section{Bibliografía}

Antonini, R. (2015). “Child language brokering”. En F. Pöchhacker (eds.) Routledge Encyclopedia of Interpreting Studies, (pp. 48-49). Abindong, Oxon: Routledge.

Collados Aís, Á. \& Fernández Sánchez, M. M. (2001). Manual de interpretación bilateral. Granada: Editorial Comares, S.L.

Crezee, I. H. M., Mikkelson, H. \& Monzón-Storey, L. (2015). Introduction to Healthcare for Spanish-speaking Interpreters and Translators. Amsterdam/Philadelphia: John Benjamins Publishing Company.

Foulquié-Rubio, A.-I. (2018). “Aproximación a la interpretación en los servicios públicos en la Región de Murcia”. En A.-I. FoulquiéRubio, M. Vargas-Urpi \& M. Fernández Pérez (eds.) Panorama de la traducción y la interpretación en los servicios públicos españoles: una década de cambios, retos y oportunidades, (pp. 137-150). Granada: Editorial Comares.

Gobierno de Canarias [Hipot-CNV] <https://www3.gobiernodecanarias.org/sanidad/scs/contenidoGenerico.jsp?idDo cument=c528d8e3-f147-11e7-9d56-c37102939259\&idCarpeta=8f94f980-d052-11e7-836b-953b40afb30b> [17/11/2017].

Gobierno de Canarias [TRADASSAN] <https://www3.gobiernodecanarias.org/sanidad/scs/contenidoGenerico.jsp?id Document=b66fed8e-f147-11e7-9d56-c37102939259\&idCarpeta=8f94f980-d052-11e7-836b-953b40afb30b > [17/11/2017]. 
Hale, S. (2012). La Interpretación Comunitaria. La interpretación en los sectores jurídico, sanitario y social. Granada: Editorial Comares, S.L.

Hale, S. (2015). “Community Interpreting”. En F. Pöchhacker (eds.) Routledge Encyclopedia of Interpreting Studies, (pp. 65-69). Abindong, Oxon: Routledge.

Hsieh, E. (2015). “Healthcare interpreting”. En F. Pöchhacker (eds.) Routledge Encyclopedia of Interpreting Studies, (pp. 177-182). Abindong, Oxon: Routledge.

ISTAC [Instituto Canario de Estadística] <http://www.gobiernodecanarias.org/istac/jaxi-istac/tabla.do > [10/05/2019].

Jiménez Ivars, A. (2012) Primeros pasos hacia la interpretación Inglés-Español. Madrid: Edelsa.

Ministerio de Industria, Energía y Turismo <https://www.mincotur.gob.es/es-es/Paginas/index.aspx> [13/06/2018].

Muñoz Martínez, R. (2010). El acceso a los medios sanitarios en la diversidad cultural. Mediación intercultural en el ámbito sanitario y terapias interculturales en salud mental, estudios de caso en Bélgica y España. Tesis Doctoral. Universidad Autónoma de Madrid.

Perdomo Cerpa, M. (1992). Firgas. Noticias de un Siglo 1835-1935. Gran Canaria: Estudigraf, S.L.

Pérez-Luzardo Díaz, J. \& Fernández Pérez, M. (2018). "La provisión de la traducción y la interpretación en los servicios públicos de Canarias: retos de un territorio insular”. En A.-I. Foulquié-Rubio, M. Vargas-Urpi \& M. Fernández Pérez (eds.) Panorama de la traducción y la interpretación en los servicios públicos españoles: una década de cambios, retos y oportunidades, (pp. 65-81). Granada: Editorial Comares.

Pérez, H. (2016). Azuaje, Barranco de (Firgas y Moya). Mi Gran Canaria. Origen y noticias de sus lugares $<$ http://toponimograncanaria.blogspot.com/search?q=azuaje $>$ [20/07/2019].

Pöchhacker, F. (2004). Introducing interpreting studies. London/New York: Routledge.

Toledano Buendía, C., Fumero, M. C. \& Díaz Galván, A. (2006). “Traducción e interpretación en los servicios públicos: situación en la comunidad autónoma de canaria". RESLA.Vol. 1., 187-202. 